\title{
Mapas Conceituais no Ensino de Bioquímica, uma Integração entre os Conceitos Científicos
}

\section{Concept Maps in Teaching of Biochemistry, an Integration between Scientific Concepts}

\author{
Denise Braga Schimidt ${ }^{*}$, Lorraine Herdy Heggendornn ${ }^{1}$, Helena de Souza Pereira ${ }^{2}$, Valéria Vieira $^{3}$, Fábio \\ Aguiar-Alves ${ }^{1,4}$

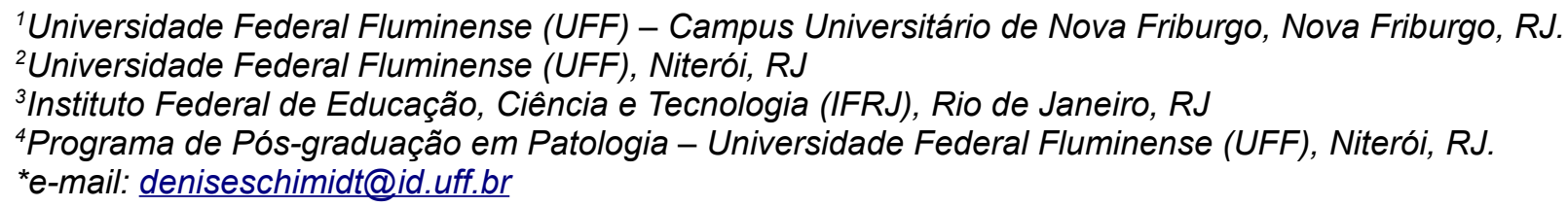

\section{Resumo}

Os conteúdos apresentados na disciplina de Bioquímica exigem conhecimentos científicos prévios e são, muitas vezes, descontextualizados e fragmentados, impedindo um significado para o aprendizado. O artigo relata a experiência com o uso de uma metodologia alternativa no ensino de Bioquímica: Mapas Conceituais. O desenho procedimental baseou-se no uso de questões objetivas com questionários conceituais antes e após a leitura do Mapa Conceitual desenvolvido a partir de tema estudado na disciplina de Bioquímica do curso de Odontologia da UFF (RJ). O tema Integração do Metabolismo, consenso entre os docentes como capaz de relacionar diferentes conceitos, foi escolhido para elaboração do Mapa. Objetivou-se demonstrar que tal ferramenta de ensino pode permitir integração de conceitos. Os resultados mostraram que, após o uso do Mapa, o número de alunos que acertou grande parte ou todas as questões propostas no questionário foi consideravelmente maior, sugerindo Aprendizagem Significativa.

Palavras-chave: Metodologias de ensino; Ensino de Bioquímica; Mapas conceituais.

\begin{abstract}
The contents of Biochemistry discipline require previous scientific knowledge and it is often disconnected and fragmented, voiding a meaningful learning. This article describes an experience with the use of an alternative methodology to teach Biochemistry: Concept Maps. The procedural design was based on the use of subject questionnaires before and after reading the Concept Map developed from a subject studied in Biochemistry during the graduation program for the dental school of UFF (RJ, Brazil). Integration of Metabolism is consensus among teachers as a topic able to effectively correlate different concepts as it has been chosen to prepare the Map. The aim of this study was to show this teaching tool that might allow concepts' integration. The results showed, after using the Map, a higher number of students getting right answers to all proposed questions, suggesting improvement in Significant Learning.
\end{abstract}

Keywords: Methodologies; Teaching Biochemistry; Concept Maps. 


\section{Introdução}

No decorrer do processo ensino-aprendizagem, quando se enfatizam atividades que favoreçam a espontaneidade do aluno, permite-se que este construa noções necessárias para a compreensão da ciência. De acordo com Moura e Vale [1], a didática atualmente utilizada em sala de aula é majoritariamente "conteudista", ou seja, a preocupação principal é quase sempre a transmissão de conteúdos. Essa didática, ainda para os autores, não considera a realidade do aluno e não se baseia nos conhecimentos que eles trazem para o local de estudo, muito menos reforça a necessidade de uma integração com esses conteúdos.

A Bioquímica, disciplina básica presente nos cursos de formação da área da saúde, de acordo com Zeni [2], é uma das que mais apresenta dificuldade de compreensão entre os alunos. Contudo, observa-se que estes apresentam dificuldades sobre os temas trabalhados nesta disciplina desde os primeiros contatos com temas relacionados ainda no ensino médio. O ensino desses conteúdos é discutido por Luz et al [3].

Não é raro, portanto, encontrar situações em que os estudantes sintam-se desinteressados, porque não conseguem perceber a relação entre os conteúdos que estão estudando. Além disso, o conteúdo curricular de Bioquímica é particularmente difícil para o aluno, principalmente por dois motivos: pelo fato de necessitar de conhecimentos científicos prévios sobre propriedades das substâncias presentes no organismo, e, de concordar que determinadas reações ocorrem, mesmo sem a visualização das interações entre as moléculas e transformações sofridas pelas mesmas, trazendo assim uma dificuldade de compreensão desses conteúdos trabalhados pelos docentes [4].

Outro problema normalmente observado em relação ao estudo de Bioquímica é que, embora se possam estudar partes individuais do conteúdo curricular em qualquer sistema, deve-se considerar que essas partes não estão isoladas do todo. Segundo Cox [5], esse fato pode ser exemplificado com o conteúdo Metabolismo, no qual seus componentes são ensinados individualmente, mas necessitam ser integrados para o entendimento da complexidade das reações e transformações bioquímicas. Desta forma, sugere-se que a visão fragmentada utilizada normalmente nas salas de aula pode justificar a dificuldade de compreensão dos alunos e a consequente dificuldade na formulação de questões diante de problemas complexos.

Sendo assim, a falta de motivação, considerada muitas vezes como uma das principais causas do desinteresse dos alunos com relação ao estudo e compreensão dos 
novos conceitos, em muitas situações, pode estar relacionada à metodologia de ensino utilizada pelo professor [6].

Possibilidades para tornar alunos mais motivados e melhorar a aprendizagem de conceitos científicos são sempre apresentadas em literaturas de educação, por exemplo, em instrumentos para a avaliação dessa compreensão ou em aulas mais dinâmicas associadas às tradicionais aulas expositivas [7]. Trabalhos como o de Mattos [8] discutem as possibilidades de os professores mudarem sua maneira de ensinar em cursos de graduação em Bioquímica e Biologia Molecular. Uma ferramenta de ensino apropriada para dinamizar as aulas teóricas pode cumprir o papel, também, de integração dos tópicos e assuntos expostos pelo professor [9-10].

A partir desse contexto se optou por utilizar como ferramenta de ensino, para integração dos conteúdos científicos e construção do conhecimento, a confecção de Mapas Conceituais, representações gráficas de conjuntos de conceitos que se relacionam.

Os Mapas Conceituais, metodologia desenvolvida principalmente por Novak, na Universidade de Cornell em 1972, baseiam-se na Teoria da Aprendizagem Significativa [12]. De acordo com tal teoria, de Ausubel et al. [11], toda aprendizagem é um processo no qual o aprendiz relaciona a nova informação com o conhecimento prévio que há no seu cognitivo. Para Novak e Cañas [12], "mapas conceituais são ferramentas gráficas para organizar e representar conhecimento. Eles incluem conceitos, usualmente dentro de círculos ou caixas de algum tipo e a indicação da relação entre os conceitos é realizada por linhas que ligam esses conceitos".

Segundo alguns autores, os Mapas Conceituais podem demonstrar o nível de aprofundamento de compreensão do sujeito durante a construção dessas representações gráficas, ou, indicar a relação entre esses conceitos [13-14]. O fato de não necessariamente implicarem sequência, temporalidade ou direcionalidade mostra a diferença existente entre os mapas conceituais e diagramas ou organogramas de fluxo [15]. De acordo com Maffra [16], durante a construção de um mapa conceitual, os conceitos são colocados dentro de uma figura geométrica, enquanto as relações entre eles seriam demonstradas através de frases de ligações representadas por linhas conectando os conceitos. Assim, os mapas conceituais permitem que o aluno organize seus conhecimentos de acordo com os antigos e novos conceitos adquiridos [4, 17-18]. 
Uma característica importante relacionada aos mapas conceituais, destacada por Grillo e Lima [19] está relacionada ao fato de que os mapas são representações pessoais, portanto, ao explicar um mapa conceitual, o autor externaliza significados, estando aí o maior valor de um mapa conceitual [20]. Dessa forma, a elaboração de mapas conceituais tanto pelo professor, quanto pelo aluno pode contribuir efetivamente para que a compreensão dos conteúdos torne-se mais clara, uma vez que diferentes pontos de vista podem ser considerados.

Embora aparentemente simples e até mesmo confundidos com esquemas, diagramas ou organogramas, os mapas conceituais têm como objetivo fazer com que determinados conceitos sejam esclarecidos e apresentem algum significado quando separados, e, principalmente quando juntos e integrados [17]. Considerado como um recurso que visa facilitar a aprendizagem de conceitos, o mapa conceitual não necessita de recursos ou equipamentos sofisticados, por isso pode ser utilizado amplamente pelos estudantes. [18-19]

Existem princípios metodológicos a serem considerados na construção dos mapas com os conceitos que devem relacionar-se de forma coerente, seguindo uma ordenação lógica, e, as palavras de enlace, que junto aos conceitos, permitem construir frases com significado lógico [20]. A estrutura do mapa deve permitir uma leitura de cima para baixo ou de baixo para cima, para que seja possível mostrar que as palavras utilizadas para a caracterização de determinado assunto se integram, evidenciando que separadamente tudo aquilo pode não fazer sentido, dependendo do assunto abordado [21]. Além disso, outra vantagem observada é a possibilidade que o aluno tem de visualizar o conteúdo adquirido no processo de ensino-aprendizagem, auxiliando, portanto, aqueles estudantes que têm memória visual [22].

Dependendo do assunto a ser tratado e da forma como se deseja explicar determinado conteúdo, o mapa conceitual pode ser organizado de uma forma que facilite o entendimento e dê mais clareza e ênfase aos tópicos que devem respeitar determinada hierarquia conceitual. Assim, quando se trabalha com confecção de mapas conceituais no auxílio do processo ensino-aprendizagem, devem-se conhecer os diferentes tipos para que seja possível uma prévia análise sobre o perfil do mapa mais adequado às respectivas necessidades [23-24].

Em relação a sua forma gráfica, os mapas conceituais podem ser construídos nos 
formatos unidimensional (conceitos dispostos de forma vertical), bidimensional (conceitos em disposições vertical e horizontal) ou tridimensional [25]. Por serem mais completos que os mapas unidimensionais, além de mais simples de serem interpretados que os mapas tridimensionais, os mapas bidimensionais são os mais utilizados [21]. Quanto a sua estrutura, os mapas conceituais podem ser classificados em três tipos principais: o tipo teia, que apresenta um conceito central, gerador de outros conceitos, os quais vão sendo inseridos à medida que se afasta do centro; tipo fluxograma, que apresenta os conceitos organizados de forma linear; e tipo hierárquico, que apresenta o conceito mais importante no início da cadeia hierárquica, e os demais relacionados a este, são inseridos de forma descendente de importância em relação ao tema esquematizado [25-26].

Nesse trabalho, com o objetivo de facilitar a aprendizagem do ensino de Bioquímica e de tentar torná-lo mais simples aos alunos, descreve-se uma experiência prática do uso de um mapa conceitual. Buscou-se analisar, por meio da utilização de um mapa conceitual, o desempenho de alunos que cursavam a disciplina de Bioquímica, comparando a capacidade de integração dos tópicos que envolvem um tema específico (Introdução ao Metabolismo) a partir de uma exposição teórica anterior do conteúdo.

\section{Procedimentos}

Este trabalho foi desenvolvido com 44 (quarenta e quatro) alunos que cursavam a disciplina de Bioquímica do curso de Odontologia da Universidade Federal Fluminense (RJ), no segundo semestre de 2011. Os alunos foram voluntários na participação desta pesquisa. Diversas estratégias para a aplicação de mapas conceituais podem ser formuladas [16]. A estratégia aqui proporcionada foi de apresentar aos alunos um mapa conceitual, sobre metabolismo, previamente construído, como trabalhado por Luchetta [28]. Na primeira etapa, os estudantes tiveram uma breve explicação sobre o que são e como são utilizados os mapas conceituais, ressaltando-se para a integração dos conceitos [29-31]. Todos os voluntários já haviam estudado o conteúdo teórico "Introdução ao Metabolismo", pertencente à disciplina de Bioquímica. Isso porque, embora os mapas conceituais possam ser usados para proporcionar uma visão geral de um tema, utilizá-los quando os alunos já têm algum conhecimento com o assunto é preferível, pois assim, os tópicos tratados serão significativos, permitindo a integração e diferenciação dos significados e conceitos em estudo [29]. 
Durante a explicação do mapa conceitual elaborado, evidenciou-se: o significado de cada conceito, o significado das palavras de enlace (palavras que relacionam os conceitos por meio de setas ou linhas) e também as relações existentes entre cada uma dessas palavras para compreensão do conteúdo em questão [26], pois como discutido por Grillo e Lima [19] e por Moreira [20] os mapas conceituais não são auto-explicativos, mesmo apresentando palavras-chave, e, por isso, ao explicá-lo, o autor acrescenta maior valor, mostrando os significados de suas representações pessoais relatadas.

A estratégia metodológica adotada foi de pesquisa quantitativa, e, do tipo estudo de caso. Considera-se estudo de caso o fator de ter ocorrido um acompanhamento de um semestre com esses alunos, sendo possível perceber o estímulo durante a aplicação desta metodologia, e, o relato sobre a mesma em diferentes etapas do semestre. Além disso, os acertos e erros nas respostas dos questionários, que continham cinco questões objetivas de conteúdo, serviram de apoio para incitar discussões ao final da leitura do segundo questionário, com intuito de estimular a compreensão dos alunos nas suas respostas antes e depois da apresentação do mapa conceitual.

A opção na construção do mapa conceitual utilizado, realizado pelo professor da disciplina e posteriormente apresentado e trabalhado junto aos alunos, ocorreu a partir da análise dos vários tipos possíveis de mapas conceituais que integram conceitos. $\mathrm{O}$ fato de a disciplina de Bioquímica, e o tema escolhido - Introdução ao Metabolismo - requererem que os tópicos utilizados para explicar conceitos tenham grande integração, e como o principal objetivo era fazer com que os alunos percebessem essa integração entre os conceitos que envolvem o metabolismo, utilizamos um mapa conceitual, tipo hierárquico, com inter-relações. Assim, tornou-se possível associar um conceito utilizado no início do mapa com outros adicionados posteriormente.

Durante a construção do mapa, preocupou-se com a forma com que os conceitos seriam apresentados aos alunos, sendo necessário enfatizar que cada conceito mantinha relação com um ou mais conceitos evidenciados. Além disso, para dar sentido às palavras escritas separadamente, explicitou-se a importância das setas utilizadas, associadas a poucas palavras, mas capazes de demonstrar e explicar a relação entre os conceitos.

Pesquisas com estratégias de capacitação e avaliação do conhecimento e/ou proficiência de mapas conceituais, de acordo com Aguiar [32], são raras na literatura. No presente estudo, elaborou-se um questionário (veja o questionário no apêndice A) para 
identificarmos a estruturação desses conceitos, observada, na aprendizagem significativa, como facilitadora do aprendizado, possível de ter ocorrido por meio do trabalho realizado com o desenvolvimento do mapa conceitual [7].

No questionário, elaborado pelos próprios autores, foram apresentadas aos alunos cinco questões objetivas sobre o tema tratado no mapa desenvolvido. As questões versavam sobre conceito de metabolismo, identificação e função dos seus principais componentes, bem como função e utilização de moléculas de armazenamento, além, da comparação entre as diferentes reações ocorridas durante o metabolismo. Os autores entendem que esta metodologia tem suas limitações, por oferecer somente indícios de aprendizagem. No entanto, a opção por tal metodologia se deve ao tempo limitado em sala de aula - apesar de se trabalhar um semestre inteiro com a turma, o conteúdo escolhido precisava ser aplicado em duas ou, no máximo três aulas.

Aplicou-se o mesmo questionário duas vezes para os 44 alunos. Nossa intenção foi de obter as respostas desses alunos antes e depois do trabalho de desenvolvimento do mapa, para obtenção de indícios da melhoria na aprendizagem pelos alunos dos conceitos trabalhados por meio do mapa conceitual. Assim, os alunos responderam o questionário antes de conhecerem o mapa, com base nos seus conhecimentos vistos em aulas teóricas sobre a Introdução ao Metabolismo, e, após a explicação do mapa conceitual, que enfatizava as relações entre os tópicos. Com base na comparação dessas respostas construímos nossa explanação.

Além disso, realizou-se um acompanhamento recente, passados mais de dois anos da pesquisa, com um grupo de alunos, cujo desempenho atingiu os $100 \%$ de acerto no segundo questionário, como será discutido nos resultados.

\section{Resultados e Discussão}

Por meio de uma aula expositiva, apresentou-se e explicou-se aos alunos o mapa conceitual sobre Introdução ao Metabolismo, do tipo hierárquico, como relatado nos procedimentos. A Figura 1 representa o mapa conceitual utilizado. Avaliou-se o efeito do contato do mapa conceitual sobre a fixação e compreensão dos conteúdos pelos alunos participantes por suas respostas aos questionários aplicados. 


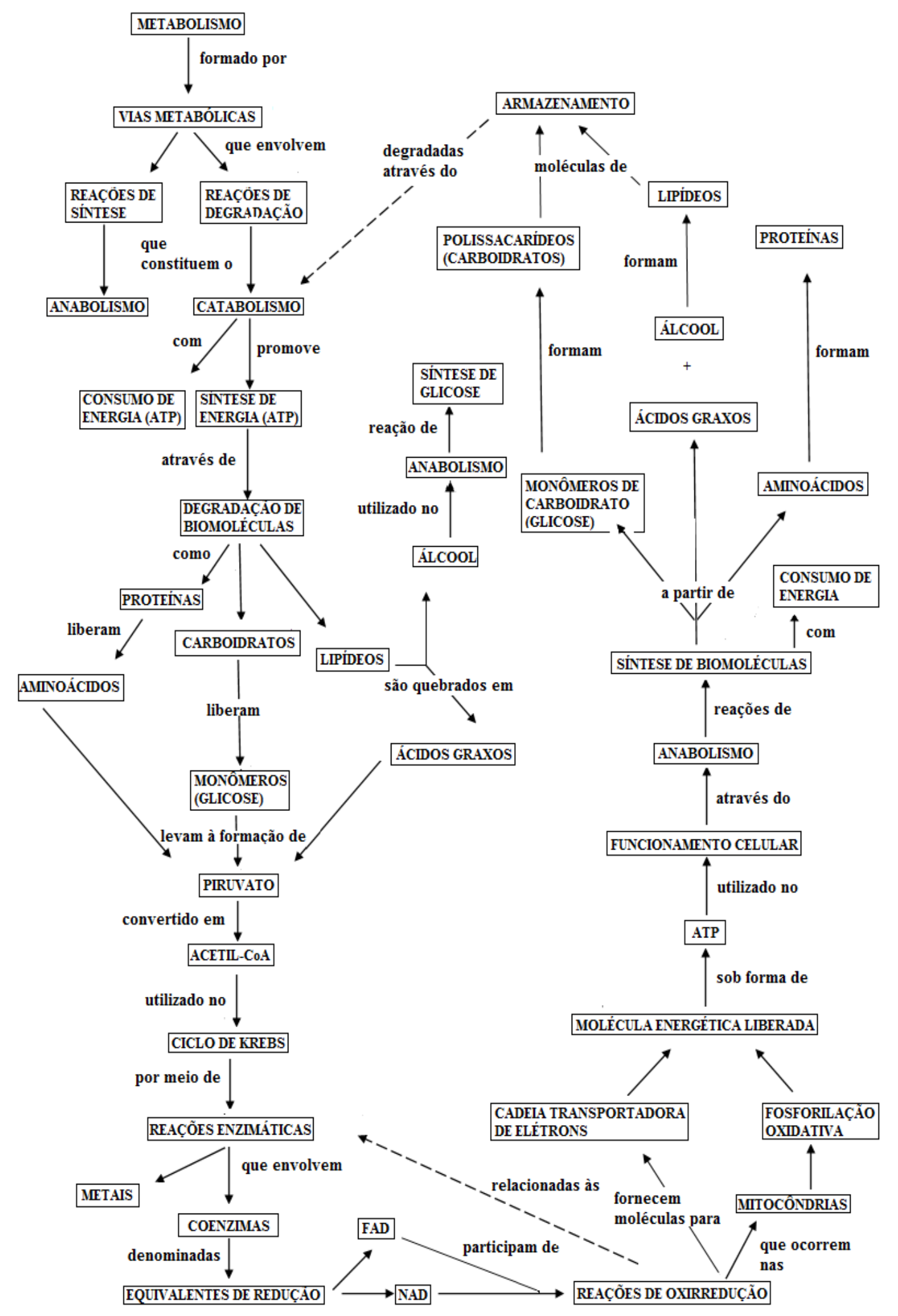

Figura 1. Mapa Conceitual relativo ao tema desenvolvido: Introdução ao Metabolismo Celular. 
A intenção na análise dos questionários foi de obter indícios se haveria algum impacto na assimilação de conteúdos após o trabalho com o mapa conceitual. Ao final da aula expositiva com o mapa, os alunos que já haviam respondido um primeiro questionário, responderam o segundo. Neste, estavam às mesmas questões do primeiro, porém, agora com a influência da visualização e trabalho realizado com o mapa conceitual.

Com relação aos questionários aplicados, optou-se por um método no qual se comparou os acertos e erros dos alunos em cada uma das questões propostas, anteriormente e posteriormente à aplicação do mapa conceitual. Desta forma, podem-se propor algumas discussões, considerando como parâmetros resposta correta da questão, resposta incorreta da questão e não responder a questão proposta, sendo os dados obtidos representados na Figura 2.
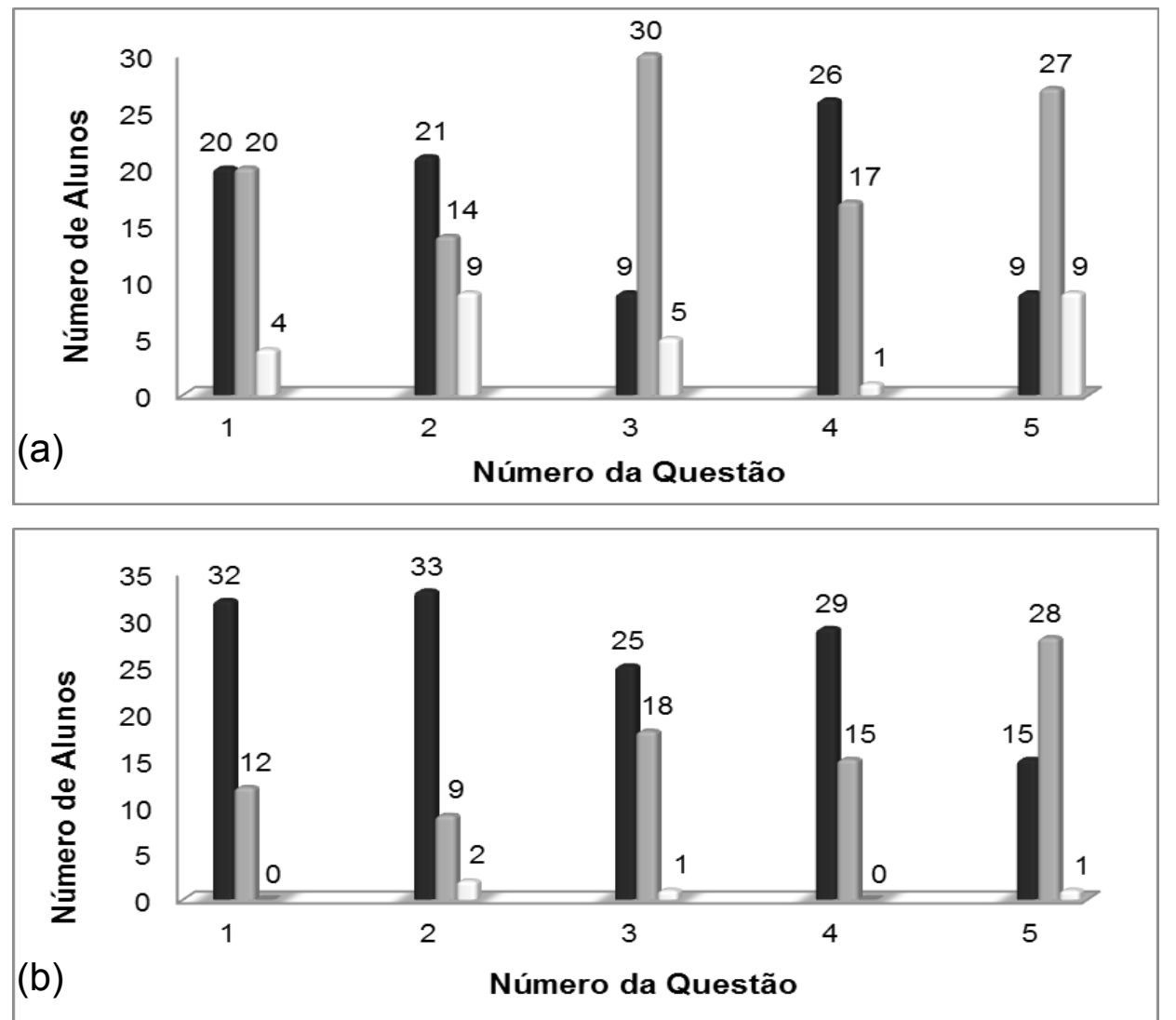

Figura 2. Gráficos que relacionam o número das questões do questionário desenvolvido (questões de 1 a 5 ) com a quantidade de alunos que as responderam corretamente (barras pretas); responderam incorretamente (barras cinzas); não responderam (barras brancas). Considerando-se os 44 alunos participantes do estudo, os dados representados se referem: (a) à primeira aplicação do questionário - ou seja, antes da utilização do mapa conceitual desenvolvido; (b) à segunda aplicação do questionário - ou seja, após a utilização do mapa conceitual desenvolvido. 
Como as respostas dos estudantes às questões dependiam diretamente da compreensão do tema tratado no mapa conceitual, principalmente da integração dos conceitos, enfatizada neste, avaliamos o fato de o aluno responder corretamente às questões como ponto positivo para a compreensão do conteúdo.

Como pode ser observado por meio da comparação entre os dados fornecidos nos gráficos representados na Figura 2, o número de acertos cresceu em todas as questões. Uma das possíveis explicações para este fato pode ser o reforço da teoria durante a aplicação do mapa conceitual, considerado como um resultado positivo para a aprendizagem porque o objetivo do trabalho foi o aperfeiçoamento da mesma. Apresentase na Tabela 1 o número de acertos dos alunos por questão presente nos questionários utilizados, comparando os erros e acertos antes e após a aplicação do mapa conceitual.

Tabela 1. Quantidade de alunos que acertou cada uma das cinco questões (1 a 5 ) ao responder o questionário fornecido antes e após a aplicação do mapa conceitual (número de acertos antes e número de acertos depois, respectivamente).

\begin{tabular}{ccccccc}
\hline & & Questão 1 & Questão 2 & Questão 3 & Questão 4 & Questão 5 \\
\hline \multirow{2}{*}{$\begin{array}{c}\text { Número de } \\
\text { acertos }\end{array}$} & Antes & 20 & 21 & 9 & 26 & 9 \\
\cline { 2 - 7 } & Depois & 32 & 33 & 25 & 29 & 15 \\
\hline
\end{tabular}

No entanto, ressalta-se para as questões 3 e 5, que tiveram número de acertos na primeira fase abaixo de $40 \%$. As mesmas que versavam, respectivamente, sobre o armazenamento de moléculas e comparação entre duas reações, apresentaram na segunda fase um maior número de acertos, sendo que a de armazenamento ultrapassou os $50 \%$, como quase todas as questões respondidas nessa fase, com exceção da quinta questão que mesmo quase dobrando o número de indivíduos que a acertaram (de 9 para 15) ainda apresentou uma porcentagem abaixo de todas as outras na segunda fase (34\%). Uma possível justificativa para o desempenho dos alunos nessa questão é que, como umas das reações especificadas (anabolismo) ocorre no início do mapa e não apresenta outros desdobramentos, diferente do que é desenhado para catabolismo, sua função poderá não ter ficado completamente esclarecida aos alunos.

Discutindo-se uma análise individual observou-se que 25 dos 44 alunos melhoraram consideravelmente seu desempenho (quando fizeram o questionário pela segunda vez aumentaram o número de acertos e não erraram nenhuma questão que tinham acertado na primeira vez); 6 alunos permaneceram com o mesmo número de 
acertos, quando fizeram o questionário pela segunda vez, acertando as mesmas questões; 10 erraram alguma questão que antes tinham acertado; 3 diminuíram o número de acertos na segunda vez em que se aplicou o questionário. Para este resultado, gostaríamos de ressaltar que mais de $50 \%$ desses alunos, grupo daqueles que aumentaram o número de acertos, possivelmente tiveram um aperfeiçoamento no processo de aprendizagem.

Outro resultado que se percebeu foi o aumento da quantidade de alunos que conseguiu acertar todas as questões. Na primeira vez em que o questionário foi aplicado, apenas 1 aluno acertou as 5 questões propostas, enquanto na segunda vez de aplicação do mesmo questionário, 6 alunos tiveram acerto em todas as questões. Esse melhoramento no desempenho dos alunos ressalta a possibilidade de um real aprendizado nesses conceitos científicos trabalhados com o mapa conceitual, visto que estudos apontam que estudantes que frequentemente usam diagramas e imagens, em vez de apenas se orientar por textos, apresentam desenvolvimento de modelos mentais de forma mais significativa [23].

No entanto, surgiu para os autores, tempos depois da aplicação deste trabalho, um questionamento sobre esses 6 alunos que acertaram todas as questões posteriormente à aplicação do Mapa Conceitual. Será que atualmente esses alunos que cursam hoje o sétimo semestre, ou seja, mais de dois anos após a pesquisa, mantém esses acertos?

Para obter essa resposta, o questionário (apêndice A) foi novamente aplicado para os seis alunos, nos quais se pode observar o seguinte desempenho: dois alunos acertaram todas as questões, três alunos erraram somente uma questão (dois erraram a de número 2 e um a de número 5), e, um aluno errou as questões 2 e 5.

Portanto, para o perfil observado nos acertos e erros do questionário pode-se discutir que a metodologia de ensino utilizada para o trabalho de conteúdos curriculares de um curso de Bioquímica no Ensino Superior foi parte integrante de um processo de amadurecimento e conexão desses conteúdos. Durante a apresentação do mapa buscouse trabalhar com uma "diferenciação progressiva" que segundo Moreira e Buchweitz [30], implica na hierarquização de conceitos mais abrangentes até os mais específicos, que quanto mais integrados, refletem melhor estruturação de conhecimento. A diferenciação progressiva é a primeira de quatro princípios essenciais da Aprendizagem Significativa, os outros três seriam: a reconciliação integrativa, a organização sequencial e a consolidação, 
respectivamente. Desta forma, a visão fragmentada dos tópicos da disciplina de Bioquímica pontuada como necessária modificação, pode ser apresentada de forma conectada por meio do mapa conceitual.

\section{Conclusões}

Torna-se muito enfática a afirmativa que o aprendizado desses alunos aperfeiçoouse com a apresentação do mapa, mas, é notório um melhor desempenho na resposta das questões objetivas dos alunos quando os mesmos tinham sido submetidos a esta didática diferenciada. Além disso, percebe-se, no contato direto com esses aprendizes, a identificação de estímulos que os levam a discutir mais em sala de aula os conceitos abordados em Introdução ao Metabolismo.

Mais enfático ainda é saber que essa assimilação, ou quem sabe aprendizado, persistiu nas concepções dos alunos mesmo passados mais de dois anos do trabalho com o Mapa Conceitual, reforçando a importância do procedimento educacional.

A experiência aqui relatada foi fruto de trabalho entre docentes e discentes dos cursos de Odontologia e Biomedicina da UFF, RJ, onde buscou-se fomentar a discussão das práticas de ensino em prol de um melhor processo ensino-aprendizagem. Como esta metodologia não se encerra em um fim, e, sim, faz parte do processo de construção, este trabalho se propõe a ter continuidade anual em diferentes turmas.

\section{Referências}

[1] Moura GRS, Vale JMF. O Ensino de Ciências na $5^{a}$ e na $6^{a}$ Séries do Ensino Fundamental. Educação em Ciências, da Pesquisa a Prática Docente (org. Roberto Nardi). São Paulo: Escrituras Editora. 2002.

[2] Zeni ALB. Conhecimento Prévio para a Disciplina de Bioquímica em Cursos da Área de Saúde da Universidade Regional de Blumenau-SC. Rev Bras Ens Bioq Biol Mol. 2010; 2 (1): 1-14.

[3] Luz MR, Oliveira GA, Da Poian AT. Glucose as the Sole Metabolic Fuel: Overcoming a Misconception Using Conceptual Change to Teach the Energy-yielding Metabolism to Brazilian High School Students. Biochem Mol Biol Educ. 2013; 41(4): 224-231.

[4] Azevedo AMP, Lazzarotto GB, Timm MI, Zaroonzio MA. Relato de uma Experiência com o Uso do Diagrama Metabólico Dinâmico Virtual do Ciclo de Krebs. Revista Renone: Novas Tecnologias na Educação (UFRGS). 2004; 1(2): 1-9.

[5] Cox JR. Enhancing Student Interactions with the Instructor and Content Using Pen- 
based Technology, YouTube Videos, and Virtual Conferencing. Biochem Mol Biol Educ. 2011; 39, (1): 4-9.

[6] Fialho NN. Os Jogos Pedagógicos como Ferramenta de Ensino. In: Congresso Nacional de Educação; 06 a 09 de outubro de 2008, Curitiba, PR. Anais do VIII Congresso Nacional de Educação (EDUCERE). 2008. p. 12298-12306. Acesso em: 27 de janeiro de 2012 . Dis ponível e m: http://www.pucpr.br/eventos/educere/educere2008/anais/trabalhos10.html.

[7] Anderson TR, Schonborn KJ. Bridging the Educational Research-teaching Practice Gap: Conceptual Understanding, Part 2: Assessing and Developing Student Knowledge, Biochem Mol Biol Educ. 2008; 36 (5): 372-379.

[8] Mattos C, Johnson M, White H, Sears D, Bailey C, Bell E. Introduction: Promoting Concept Driven Teaching Strategies in Biochemistry and Molecular Biology. Biochem Molec Biol Educ. 2013; 41 (5): 287-288.

[9] Santos VT, Anacleto AC. Monitorias como Ferramenta Auxiliar para Aprendizagem da Disciplina Bioquímica: Uma Análise no UNILESTE-MG. Rev Bras Ens Bioq Biol Mol. 2007; 1: E1-E8.

[10] V. Oenning, J.M.P. Oliveira (2011), Dinâmicas em sala de aula: envolvendo os alunos no processo de ensino, exemplo com os mecanismos de transporte da membrana plasmática. Rev. Bras. de Ens. de Bioq. e Biol. Molec, article 3, ed. 01/2011.

[11] Ausubel DP, Novak JD, Hanesian H. Educational Psychology: a Cognitive View. 2. ed. New York, 2 ed. 733p; 1978.

[12] Novak JD, Cañas AJ. The Theory Underlying Concept Maps and How to Construct and Use Them, Technical Report IHMC CmapTools 2006-01 Rev 01-2008, Florida Institute for Human and Machine Cognition. 2008. Acesso em 27 de janeiro de 2011. Disponível em:

http://cmap.inmc.us/Publications/ResearchPapers/TheoryUnderlyingConceptMaps.pdf.

[13] L. Roberts (1999), Using concept maps to measure statistical understanding, Int. J. Math. Educ. Sci. Technol. 20, (5), 707-717.

[14] Novak JD, Gowin DB. Learning How to Learn. New York: Cambridge: University Press. 1984.

[15] Gomes AP. Mapas Conceituais e o Ensino da Medicina. Rev Bras Edu Med. 2011; 35 (2): 275-282.

[16] Maffra SM. Mapas Conceituais como recurso facilitador da Aprendizagem Significativa - uma abordagem prática. Dissertação de Mestrado do Programa de Pós Graduação de Ensino de Ciências, IFRJ - 2011

[17] J.D. Novak (1998), Learning, Creating, and Using Knowledge: Concept Maps as Facilitative Tools for Schools and Corporations, Mahwah, N.J.,Lawrence Erlbaum and Assoc., 317p.

[18] White BT, Bolker ED. Interactive Computer Simulations of Genetics, Biochemistry and Molecular Biology. Biochem Mol Bio Educ. 2008; 36 (1): 77-84. 
[19] Grillo M, Lima MRV. Mapas Conceituais e sua Utilização na Educação. 2011. Acesso e m 01 de fevereiro de 2012. Disponível em: http://www.colegiosantissima.com.br/site/pages/formacaocontinuada/files/02.07.11/mapas conceituais.pdf.

[20] Moreira MA. Mapas Conceituais e Aprendizagem Significativa. Porto Alegre: Instituto de Física, Universidade Federal do Rio Grande do Sul (adaptado e atualizado, em 1997, de Moreira, MA. Mapas conceituais e Aprendizagem significativa. O Ensino, Pontevadra / Espanha e Braga / Portugal, 23-28, 1988: 87 - 95).

[21] Moreira MA. Mapas Conceituais - Instrumentos Didáticos de Avaliação e Análise de Currículo. São Paulo. 1987.

[22] Ruiz-Moreno L, Sonzogno MC, Batista SHS, Batista NA. Mapa Conceitual: Ensaiando Critérios de Análise. Ciência e Educação. 2007; 3 (13): 453-463.

[23] Costamagna AM. Innovaciones Didácticas: Mapas Conceptuales como Expresión de Procesos de Interrelación para Evaluar la Evolución del Conocimiento de Alumnos Universitarios. Enseñanza de las Ciencias. 2001; 19 (2): 309-318.

[24] Akinsanya C, Williams M. Concept Mapping for Meaningful Learning. Nurse Educ Today, Anglia Polytechnic University. 2004; 24 (1): 41-46. Acesso em 27 de novembro de 2011. Disponível em: http://www.ncbi.nlm.nih.gov/pubmed/14690643.

[25] Freitas Filho JR. Mapas conceituais: estratégia pedagógica para construção de conceitos na disciplina química orgânica. Ciências \& Cognição. 2007; 12: 86-95.

[26] Tavares R. Construindo Mapas Conceituais. Ciência e Cognição. 2007; 12: 72-85. Acesso em: 27 de novembro de 2011. Disponível em: http://www.cienciasecognicao.org/.

[27] Moreira MA. Mapas Conceituais e Aprendizagem Significativa. Cadernos de Aplicação, 11 (2): 143 -156, 1998.

[28] Luchetta LH. Mapas Conceituais na Prática Pedagógica. IX Congredso Nacional de Educação e III Encontro Brasileiro de Psico Pedagogia, PUCPR, 2009

[29] Santos VJSV, Oliveira EA, Garzon JCV. Desenvolvimento e Avaliação de uma Ferramenta para Diagnóstico de Literacia Visual Contextualizada no Ensino de Metabolismo. Rev Ens Bioq. 2013; 1: 54-88.

[30] G.A.B. Lima (2004), Mapa conceitual como ferramenta para organização do conhecimento em sistema de hipertextos e seus aspectos cognitivos. Perspectiva em Ciência da Informação. 9 (2), 134-145.

[31] Moreira MA, Buchweitz B. Novas Estratégias de Ensino e Aprendizagem: os Mapas Conceptuais e o Vê Epistemológico. Plátano Edições Técnicas. 1993.

[32] Aguiar JG. Desenvolvimento e Validação de um Questionário para Avaliar o Nível de Conhecimento dos Alunos sobre Mapas Conceituais. Dissertação de Mestrado em Ensino de Ciências, USP, 2012. 
Apêndice A. Questionário aplicado aos alunos participantes do estudo para avaliação do efeito do mapa conceitual utilizado.

\section{QUESTÄO 01:}

A partir do conceito de metabolismo, pode-se afirmar que:

I. O anabolismo é uma subdivisão do metabolismo e relaciona-se com reações de síntese que envolvem a liberação de energia (ATP), podendo ser classificado como convergente.

II. A partir de aminoácidos, monossacarídeos e ácidos graxos, por reações que envolvem o catabolismo, ocorre gasto de ATP para que biomoléculas, como proteínas, polissacarídeos e lipídeos sejam sintetizadas.

III. Pela degradação de biomoléculas, as reações enzimáticas do catabolismo promovem a obtenção de compostos menores, que são utilizados pelo organismo como forma de obtenção de piruvato, o que evidencia que o catabolismo é convergente.

IV. Reações do anabolismo são as que promovem a síntese de compostos que podem ser armazenados pelo organismo, sendo para isso, necessário o gasto de energia (ATP).

Estão corretas, as afirmativas:
a) I, II, III e IV
b) I e III apenas
c) II e III apenas
d) II, III e IV apenas
e) III e IV apenas 


\section{QUESTÄO 02:}

Os equivalentes de redução podem ser utilizados no metabolismo pelas reações da fosforilação oxidativa, contribuindo para a ocorrência da cadeia transportadora de elétrons. Com base nessa afirmação, marque a alternativa correta:

a) Polissacarídeos provenientes de reações do catabolismo, no citosol, participam da fosforilação oxidativa e cadeia transportadora de elétrons quando se associam aos equivalentes de redução, através do consumo de ATP.

b) No interior das mitocôndrias, através de reações de oxirredução, moléculas de NAD e FAD participam da cadeia transportadora de elétrons, e assim, ocorre a síntese de ATP.

c) As reações de fosforilação oxidativa e a cadeia transportadora de elétrons consomem diretamente moléculas de ATP, e assim, o objetivo final do catabolismo é apenas a degradação de compostos, não contribuindo para a síntese de energia.

d) O acetil-CoA, molécula proveniente do catabolismo, é utilizado nas mitocôndrias através do ciclo de Krebs, o que promove a fosforilação oxidativa e cadeia transportadora de elétrons, no citosol, para que se obtenha ATP.

e) Através do consumo de ATP (por reações do catabolismo), as coenzimas participam da fosforilação oxidativa e cadeia transportadora de elétrons, fornecendo ADP, utilizado no funcionamento celular.

\section{QUESTÄO 03:}

Algumas moléculas podem ser armazenadas no interior de células para que forneçam ATP ao organismo, quando necessário. A partir dessa afirmativa, marque a opção correta:

a) Polissacarídeos, lipídeos e proteínas são utilizados como moléculas de armazenamento celular, após serem sintetizadas em reações que envolvem o anabolismo.

b) Os lipídeos são os únicos compostos utilizados pelo organismo como forma de armazenamento energético nas células.

c) Apenas polissacarídeos e lipídeos, provenientes de reações do anabolismo, podem ser utilizados como moléculas de reserva energética pelas células.

d) Polissacarídeos, lipídeos e proteínas são utilizados como compostos de armazenamento celular, depois de sintetizadas em reações do catabolismo.

e) As moléculas de armazenamento (polissacarídeos e lipídeos) são degradadas através do anabolismo para ao final, fornecer ATP (energia para o funcionamento celular). 


\section{QUESTÄO 04:}

Leia as seguintes afirmativas:

I. Reações do anabolismo e catabolismo ocorrem independentemente uma das outras, portanto, anabolismo e catabolismo, apesar de constituírem o metabolismo, não se relacionam.

II. As reações do catabolismo são convergentes (em direção à formação de acetil-CoA) para promover a síntese de energia, enquanto o anabolismo apresenta-se divergente (pode partir do acetil-CoA para a formação de macromoléculas).

III. As mitocôndrias são as principais organelas envolvidas diretamente com a síntese de ATP, através da fosforilação oxidativa e cadeia transportadora de elétrons, principalmente.

Considerando-as verdadeiras (V) ou falsas (F), marque a opção correta:
a) $F, F, V$
b) $V, F, V$
c) $F, V, V$
d) $V, V, F$
e) $F, V, F$

\section{QUESTÄO 05:}

Com relação ao anabolismo e catabolismo, marque a opção incorreta:

a) A relação entre anabolismo e catabolismo pode ser evidenciada pela degradação de moléculas de armazenamento provenientes do anabolismo, por meio do catabolismo.

b) Através do anabolismo, moléculas de armazenamento, provenientes do catabolismo, são utilizadas como forma de obtenção de energia para o funcionamento celular.

c) O ATP produzido através do catabolismo pode ser utilizado para as reações de síntese do anabolismo, o que mostra a integração entre as duas vias.

d) O objetivo das reações do anabolismo é promover a síntese de biomoléculas de armazenamento para a utilização pelo organismo quando a quantidade de ATP disponível não for suficiente para manter o funcionamento celular.

e) Mesmo sendo vias metabólicas contrárias, anabolismo e catabolismo se completam e mantêm o funcionamento celular adequado. 